\title{
Entropic force approach to noncommutative Schwarzschild black holes signals a failure of current physical ideas
}

\author{
S. Hamid Mehdipour* \\ Islamic Azad University, Lahijan Branch, P. O. Box 1616, Lahijan, Iran
}

\begin{abstract}
Recently, a new perspective of gravitational-thermodynamic duality as an entropic force arising from alterations in the information connected to the positions of material bodies is found. In this paper, we generalize some aspects of this model in the presence of noncommutative Schwarzschild black hole by applying the method of coordinate coherent states describing smeared structures. We implement two different distributions: (a) Gaussian and (b) Lorentzian. Both mass distributions prepare the similar quantitative aspects for the entropic force. Our study shows, the entropic force on the smallest fundamental unit of a holographic screen with radius $r_{0}$ vanishes. As a result, black hole remnants are unconditionally inert even gravitational interactions do not exist therein. So, a distinction between gravitational and inertial mass in the size of black hole remnant is observed, i.e. the failure of the principle of equivalence. In addition, if one considers the screen radius to be less than the radius of the smallest holographic surface at the Planckian regime, then one encounters some unusual dynamical features leading to gravitational repulsive force and negative energy. On the other hand, the significant distinction between the two distributions is conceived to occur around $r_{0}$, and that is worth of mentioning: at this regime either our analysis is not the proper one, or non-extensive statistics should be employed.
\end{abstract}

PACS: 04.70.Dy, 04.50.Kd, 05.70.-a, 02.40.Gh

Key Words: Black Hole Thermodynamics, Noncommutative Geometry, Holographic Screens, Entropic Force

*mehdipour@liau.ac.ir 


\section{Introduction}

The exploration of thermal radiation from black holes has mainly emerged a close relationship between black hole physics and thermodynamics [1]. One striking clue for the nature of gravity comes from the profound investigation of black hole thermodynamics due to the fact that it is possible to provide a physical resemblance between spacetimes including horizons and the notions of temperature or entropy. In addition, a quantum theory of gravity tells us that the black hole entropy could be related to a number of microscopic states. Therefore, detailed studies of the black hole entropy may have important implications for a complete theory of quantum gravity. This is the main reason why the origin of the black hole entropy requires to be perceived at the fundamental level. In 1995, Jacobson exhibited that the Einstein equations are acquired from the laws of thermodynamics [2]. Padmanabhan also used the argument of equipartition energy of horizons to prepare a thermodynamic perspective of gravity [3]. Recently, Verlinde has proposed an emergent phenomenon for the origin of Newtonian gravity and general relativity [4]. This theory implies that gravitational interaction arises from the statistical behavior of microscopic degrees of freedom encoded on a holographic screen and can be described as a kind of entropic force, related to the information that is stored on the holographic surfaces. The idea of entropic force in different cases has been investigated by many authors [5]. Also, there are some comments on the entropic gravity scenario which indicate some short comings of this scenario in addition to its open problems [6], they can appear as the topic of a new debate.

If the gravitational force is entropic and entropy couples the emergent picture of gravity with the fundamental microstructure of a quantum spacetime, then in Verlinde's formalism we should investigate the microscopic scale effects by using tools such as noncommutative gravity for the interpretation of the microscopic structure of a quantum spacetime. Perhaps one way of observing noncommutativity is through assessing its influences on the properties of black holes. Nicolini et al [7] in a new conceptual approach to noncom-

mutative gravity, based on coordinate coherent state formalism, have improved the short distance behavior of point-like structures. In their method, curvature singularities which appear in general relativity, can be eliminated. They have demonstrated that black hole evaporation process should be stopped when a black hole reaches a minimal mass. This

minimal mass, named black hole remnant, is a result of the existence of a minimal observable length. This approach, which is the so-called noncommutative geometry inspired 
model, via a minimal length caused by averaging noncommutative coordinate fluctuations [8] cures the curvature singularity in black holes. In fact, the curvature singularity at the origin of black holes is substituted for a regular deSitter core. Accordingly, the ultimate phase of the Hawking evaporation as a novel thermodynamically steady state comprising a non-singular behaviour is concluded (for more details, see [9]). It must be noted that, generally, it is not required to consider the length scale of the coordinate noncommutativity to be the same as the Planck length. Since, the noncommutativity influences appears on a length scale connected to that region, they can behave as an adjustable parameter corresponding to that pertinent scale.

In this paper, we use noncommutative geometry inspired model to combine the microscopic structure of spacetime with the entropic description of gravity because the concept of entropy has a profound relationship with the quantum spacetime structures. The layout of the paper is as follows. We begin in Sec. 2 by outlining the entropic force approach. In Sec. 3, the entropic force in the presence of noncommutative Schwarzschild black hole is derived by considering the effect of smearing of the particle mass as Gaussian and Lorentzian distributions. Finally, a summary and the conclusion follows in Sec. 4.

\section{Entropic Force Approach}

In this section we briefly investigate Verlinde's approach. Afterwards, by considering the case of noncommutative geometry inspired Schwarzschild black hole, we try to improve the expression of black hole's entropic force by taking into account the noncommutative corrections at the scale which noncommutativity influences set in. In order to obtain the temperature from an entropic force in general relativity, we first consider a static background with a global timelike Killing vector $\xi^{\mu}$. To define a foliation of space, and viewing the holographic screens $\Omega$ as surfaces of constant redshift, we write the potential $\phi$ as $[4]+$

$$
\phi=\frac{1}{2} \log \left(-\xi^{\mu} \xi_{\mu}\right)
$$

where $\mu=0,1,2,3$. Note that $e^{\phi}$ indicates the redshift factor and it makes a connection between the local time coordinate and the reference point with $\phi=0$ at infinity.

The four acceleration $a^{\mu}$, for a particle that is placed very adjacent to the screen can

\footnotetext{
${ }^{\dagger}$ In this work natural units are used with the following definitions: $\hbar=c=G=k_{B}=1$.
} 
be written as

$$
a^{\mu}=-\nabla^{\mu} \phi
$$

As can be seen, the acceleration is perpendicular to the holographic screen. Then, with

defining the normal vector $N^{\mu}=\frac{\nabla^{\mu} \phi}{\sqrt{\nabla^{\nu} \phi \nabla_{\nu} \phi}}$, the local temperature on the screen is achieved by

$$
T=-\frac{1}{2 \pi} e^{\phi} N^{\mu} a_{\mu}=\frac{1}{2 \pi} e^{\phi} \sqrt{\nabla^{\mu} \phi \nabla_{\mu} \phi} .
$$

The change in entropy for a test particle with mass $m$ at fixed position close to the screen equals

$$
\nabla_{\mu} S=-2 \pi m N_{\mu} .
$$

The entropic force is now found to have the form

$$
F_{\mu}=T \nabla_{\mu} S=-m e^{\phi} \nabla_{\mu} \phi .
$$

The above expression is indeed the relativistic analogue of the second law of Newton $F=m a$. The redshift factor $e^{\phi}$ is added due to the fact that the gravitational force is measured with respect to the reference point at infinity.

Let us now suppose that the energy $E$ associated with the mass $M$ (assumedly larger than the test particle of mass $m$ and is located at the origin of the coordinate as the source) is distributed on a closed surface of constant redshift $\phi$. On this screen, $N$ bits of information are stored and the holographic information from mass $M$ is encoded as $d N=d A$ [10], where $A$ is the area of the screen. The energy on the screen obeys thermal equipartition,

$$
E=\frac{1}{2} \int_{\Omega} T d N=\frac{1}{4 \pi} \int_{\Omega} e^{\phi} \nabla \phi d A .
$$

This result is in agreement with the Gauss's theorem.

\section{Noncommutative Geometry Inspired Model: Gaus- sian and Lorentzian Distributions}

We now consider the metric of noncommutative Schwarzschild black hole. According to Refs. [7, 9], the mass density of a static, asymptotically flat, spherically symmetric, particle-like gravitational source cannot be a delta function distribution but will be given by a Gaussian distribution of minimal width $\sqrt{\theta}$ as follows

$$
\rho_{\theta}(r)=\frac{M}{(4 \pi \theta)^{\frac{3}{2}}} e^{-\frac{r^{2}}{4 \theta}} \text {. }
$$


The metric of the Einstein equations connected to these smeared mass Gaussian function sources is taken as the form

$$
d s^{2}=-h(r) d t^{2}+h(r)^{-1} d r^{2}+r^{2} d \Omega^{2},
$$

where $h(r)=\left(1-\frac{2 M_{\theta}}{r}\right)$, and $d \Omega^{2}=d \vartheta^{2}+\sin ^{2} \vartheta d \varphi^{2}$ is the line element on the 2dimensional unit sphere. The smeared mass distribution $M_{\theta}$ is given by

$$
M_{\theta}=\int_{0}^{r} \rho_{\theta}(r) 4 \pi r^{2} d r=M\left[\mathcal{E}\left(\frac{r}{2 \sqrt{\theta}}\right)-\frac{r}{\sqrt{\pi \theta}} e^{-\frac{r^{2}}{4 \theta}}\right] .
$$

$\mathcal{E}(x)$ is the Gaussian error function defined as $\mathcal{E}(x) \equiv \frac{2}{\sqrt{\pi}} \int_{0}^{x} e^{-t^{2}} d t$. In the commutative limit, $\frac{r}{\sqrt{\theta}} \rightarrow \infty$, the Gaussian error function tends to one and the other term will exponentially be reduced to zero. Thus, we get $M_{\theta} \rightarrow M$. By using the Killing equation $\partial_{\mu} \xi_{\nu}+\partial_{\nu} \xi_{\mu}-2 \Gamma_{\mu \nu}^{\lambda} \xi_{\lambda}=0$, and the condition of static spherical symmetry $\partial_{0} \xi_{\mu}=\partial_{3} \xi_{\mu}=0$, and also the infinity condition $\xi_{\mu} \xi^{\mu}=-1$, the timelike Killing vector of the noncommutative Schwarzschild black hole is found to be

$$
\xi_{\mu}=(-h(r), 0,0,0)
$$

that is equal to zero at the event horizon.

According to Eqs. (11)-(3), the acceleration on the spherical holographic screen with radius $r$ is computed as

$$
a^{\mu}=(0,2 \pi T, 0,0) .
$$

The above equation denotes that the screen carries a temperature in the following form (using Eq. (3) ) :

$$
T=\frac{1}{4 \pi} \frac{d h(r)}{d r}=\frac{M}{2 \pi r^{2}}\left[\mathcal{E}\left(\frac{r}{2 \sqrt{\theta}}\right)-\frac{r^{3}+2 \theta r}{2 \sqrt{\pi \theta^{3}}} e^{-\frac{r^{2}}{4 \theta}}\right] .
$$

It should be noted that the local temperature on the event horizon is the same as the Hawking temperature, i.e., $\left.T\right|_{r=r_{H}}=T_{H}$ [7, 9] (see also [11]).

The energy on the screen can be written in terms of the smeared mass distribution as

$$
E=2 \pi r^{2} T=M_{\theta}-\frac{M r^{3}}{2 \sqrt{\pi \theta^{3}}} e^{-\frac{r^{2}}{4 \theta}} .
$$

Finally, the modified Newtonian force law as the entropic force in the presence of the noncommutative Schwarzschild black hole becomes

$$
F=\sqrt{g^{\mu \nu} F_{\mu} F_{\nu}}=\frac{m M_{\theta}}{r^{2}}-\frac{m M r}{2 \sqrt{\pi \theta^{3}}} e^{-\frac{r^{2}}{4 \theta}}
$$


where $F_{\mu}=\left(0, \frac{m}{2 \sqrt{h(r)}} \frac{d h(r)}{d r}, 0,0\right)$. We note that, in the limit of $\theta$ going to zero, one recovers the conventional results for the acceleration, the local temperature, the energy, and the entropic force on the screen, respectively, as follows [12]:

$$
a^{\mu}=\left(0, \frac{M}{r^{2}}, 0,0\right), \quad T=\frac{M}{2 \pi r^{2}}, \quad E=M,
$$

and

$$
F=\frac{m M}{r^{2}}
$$

Eq. (16) is just the Newton force for the Schwarzschild black hole.

Now, as an important remark, we will show that the essential aspects of the noncommutativity approach are not specifically sensitive to the Gaussian nature of the smearing used by the authors in Ref. [7]. If we had chosen a different form for the smeared mass distribution, the general properties would be directed to wholly similar results to those above. For example, we consider a Lorentzian distribution of particle-like gravitational source as follows

$$
\rho_{\theta^{\prime}}(r)=\frac{M \sqrt{\theta^{\prime}}}{\pi^{2}\left(r^{2}+\theta^{\prime}\right)^{2}}
$$

Here the noncommutativity parameter, $\theta^{\prime}$, is not exactly the same as $\theta$. The smeared mass distribution $M_{\theta^{\prime}}$ is now given by

$$
M_{\theta^{\prime}}=\int_{0}^{r} \rho_{\theta^{\prime}}(r) 4 \pi r^{2} d r=\frac{2 M}{\pi}\left[\tan ^{-1}\left(\frac{r}{\sqrt{\theta^{\prime}}}\right)-\frac{r \sqrt{\theta^{\prime}}}{r^{2}+\theta^{\prime}}\right] .
$$

The Lorentzian smeared mass, $M_{\theta^{\prime}}$, has the same confining properties and is totally similar to the Gaussian smeared mass, $M_{\theta}$, qualitatively. In the limit $\theta^{\prime} \rightarrow 0$, we get $M_{\theta^{\prime}} \rightarrow M$. The local temperature on the screen immediately reads

$$
T=\frac{M}{\pi^{2} r^{2}}\left[\tan ^{-1}\left(\frac{r}{\sqrt{\theta^{\prime}}}\right)-\frac{r \sqrt{\theta^{\prime}}}{r^{2}+\theta^{\prime}}-\frac{2 r^{3} \sqrt{\theta^{\prime}}}{\left(r^{2}+\theta^{\prime}\right)^{2}}\right] .
$$

Using Eq. (18), we obtain

$$
T=\frac{M_{\theta^{\prime}}}{2 \pi r^{2}}-\frac{2 M r \sqrt{\theta^{\prime}}}{\pi^{2}\left(r^{2}+\theta^{\prime}\right)^{2}}
$$

Eventually, the energy and the entropic force on the screen are given by, respectively:

$$
E=2 \pi r^{2} T=M_{\theta^{\prime}}-\frac{4 M r^{3} \sqrt{\theta^{\prime}}}{\pi\left(r^{2}+\theta^{\prime}\right)^{2}}
$$




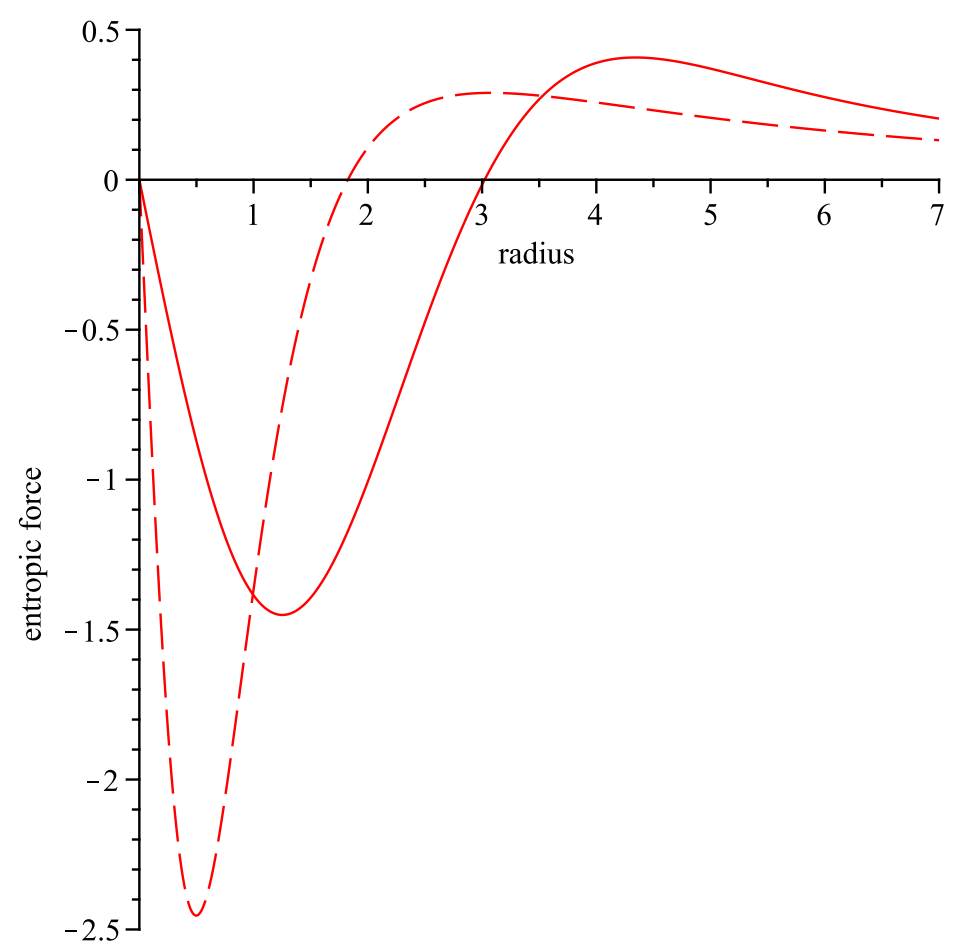

Figure 1: The entropic force as a function of radius. The solid line, corresponds to the Gaussian smearing and the dashed line, corresponds to the Lorentzian smearing. We set $m=1.0$, and $M=10.0$. For plotting the figure thus preserving the integrity of the outcomes, we set the value of the noncommutativity parameter equal to unity in both Gaussian and Lorentzian profiles; $\theta=\theta^{\prime}=1$.

and

$$
F=\frac{m M_{\theta^{\prime}}}{r^{2}}-\frac{4 m M r \sqrt{\theta^{\prime}}}{\pi\left(r^{2}+\theta^{\prime}\right)^{2}}
$$

In order to facilitate comparison between Gaussian and Lorentzian profiles, the results for the numerical solution of such noncommutative entropic force as a function of radius in both Gaussian and Lorentzian distributions (Eqs. (14) and (22)) are depicted in Fig. 1 . In both situations (Gaussian and Lorentzian) a maximum $F$ happens at relatively small $r$. As can be seen from the figure, within the noncommutative geometry inspired model, the entropic force of the black hole grows with the reduction of the radius of the spherical holographic screen until it reaches to a maximum definite value (does not diverge at all) and then falls down to zero at the minimal nonzero value of the radius, $r_{0}$. The $r_{0}$ value is seen to be nearly similar in two distributions. The minimal nonzero radius for the case of Gaussian distribution is approximately equal to $3.0 \sqrt{\theta}$, and in the case of Lorentzian distribution one has $r_{0} \approx 1.8 \sqrt{\theta^{\prime}}$. Hence, most of the results that we attained for the Gaussian case at least in asymptotic values of $r$, remain valid if we choose the other case of probability distribution of smeared matter. 
As Fig. 1 obviously shows the entropic force on the holographic screen with radius $r_{0}$ is zero. This is a significant result. The physical description of the $r_{0}$ is the radius of the smallest holographic surface which can not be probed by a test particle that is located within some distance from the source. We have seen that the standard formulation of Newtonian gravity is contravened at very short distance systems. In other words, when a test mass $m$ has a distance $r_{0}$ from the source mass, it cannot perceive any gravitational interactions. This phenomenon contravenes the existence of gravitational interactions in an inert black hole remnant [13]. Black hole remnants as essential entities are widely accepted in quantum gravity literature when quantum gravitational fluctuations emerge. For example, when generalized uncertainty principle is taken into consideration, the total decay of the black hole through radiation is prohibited, and we have massive, but inert black hole remnants with only gravitational interactions. Our approach clearly exhibits inert black hole remnants with absolutely no gravitational interactions. On the other hand, the equivalence principle of general relativity, which refers to the equivalence of gravitational and inertial mass, is violated because it is now possible to find a distinction between them. In fact, the gravitational mass in the remnant size does not emit any gravitational field, therefore it is experienced to be zero, contrary to the inertial mass $\ddagger$. In spite the fact that it is feasible to suppose both entropic gravity and noncommutative geometry are true but in the case of short distances one expects a contravention of the equivalence principle. In other words, it is possible that when one comes close to the $r_{0}$ one deviates from general relativity. Therefore, it is predictable that the equivalence principle is violated at some small scale (maybe the Planck scale) due to the combination of entropic gravity and noncommutative geometry. Indeed there is a no-go theorem for noncommutative Schwarzschild spacetime in Verlinde's approach to entropic force. Otherwise, as mentioned one would violate the equivalence principle.

In the case of $r<r_{0}$, one encounters some unusual dynamical features leading to negative entropic force, i.e. gravitational repulsive force (an exotic phenomenon, e.g. at the Planckian regime). This means that if $r$ is extremely small, as the mass $m$ approaches the screen, the decrease in screen entropy will generate a repulsive force. However, we really should not trust the details of our modeling when $r<r_{0}$. Most of the distinctions

\footnotetext{
${ }^{\ddagger}$ It should be noted that there have been other schemes which violate the equivalence principle such as the quantum phenomenon of neutrino oscillations [14, comparing Hawking radiation to Unruh radiation 15, and an examination of entropic picture of Newton's second law for the case of circular motion [16] (somewhat more related to the present work).
} 
between the Gaussian and Lorentzian profiles appear just in this extreme region. In the region that noncommutativity effects begin to be sensed exactly, the detailed nature of the sharpened mass distribution is not indeed being inspected. As a matter of fact it is possible for values $r \leq r_{0}$. But, it is important to demonstrate that according to the original work proposed by Nicolini et al [7] (for a review see [9]), concerning the thermodynamics of the noncommutative inspired black holes, for $M<M_{0}$ there is no solution for $g_{00}\left(r_{H}\right)=0$ and no horizon occurs, where $M_{0}$ is the minimal mass corresponding to an extremal black hole with one degenerate horizon in $r_{0}$. So, there should be a cut-off in the entropic force graph at some finite $r$, namely $r_{0}$. In fact, if $r<r_{0}$ or the original mass is less than the minimal mass $M_{0}$, there cannot be a black hole. Accordingly for $r<r_{0}$ we cannot speak of an event horizon and no temperature can be defined, so the final zero temperature configuration can be considered a black hole remnant. This means that when the black hole reaches the extremal configuration with a minimal mass, the temperature is zero and the Hawking emission abruptly stops.

To clarify the issue even more we show the numerical evaluations of Eqs. (13) and (21) (the energy versus the radius in both Gaussian and Lorentzian distributions) in Fig. 2 , This figure clearly exhibits that in the limit $r \gg \sqrt{\theta}$, the energy on the screen is constant. The appearance of the black hole remnant can also be seen as we approach regions with smaller screen radii; this corresponds to Fig. 1. Hence, there is also a same reason for the energy case as mentioned above for the entropic force. Furthermore, the case of $E<0$ is nonphysical and a finite cut-off is reasonable, therefore one can make the requirement that $E \geq 0$. Thus, if one considers the screen radius to be less than the radius of the smallest holographic surface at the Planckian regime, then one encounters some unusual dynamical features leading to negative entropic force and negative energy. In a recent paper [17], we have reported some results about exotic thermodynamical treatment for Planck size black hole evaporation, e.g. negative temperature, negative entropy, anomalous specific heat, and etc., which may reflect the need for a fractal nature of spacetime at very short distances. Theories such as E-infinity [18] and scale relativity [19] which are founded upon fractal structure of spacetime may provide a suitable framework to treat thermodynamics of these very short distance systems. On the other hand, in quantum gravity regime, not only the geometry containing a black hole is not truly static but also is surely dynamic because black holes are accurately considered as highly excited states. In other words, application of ordinary thermodynamics to situations such as Planck scale black hole seems to be impossible. Due to non-extensive and non-additive nature of such systems, 
one should apply non-extensive formalism such as Tsallis thermodynamics [20]. It seems that applying the Tsallis thermodynamics into Verlinde's derivation is a fascinating open problem $\S$.

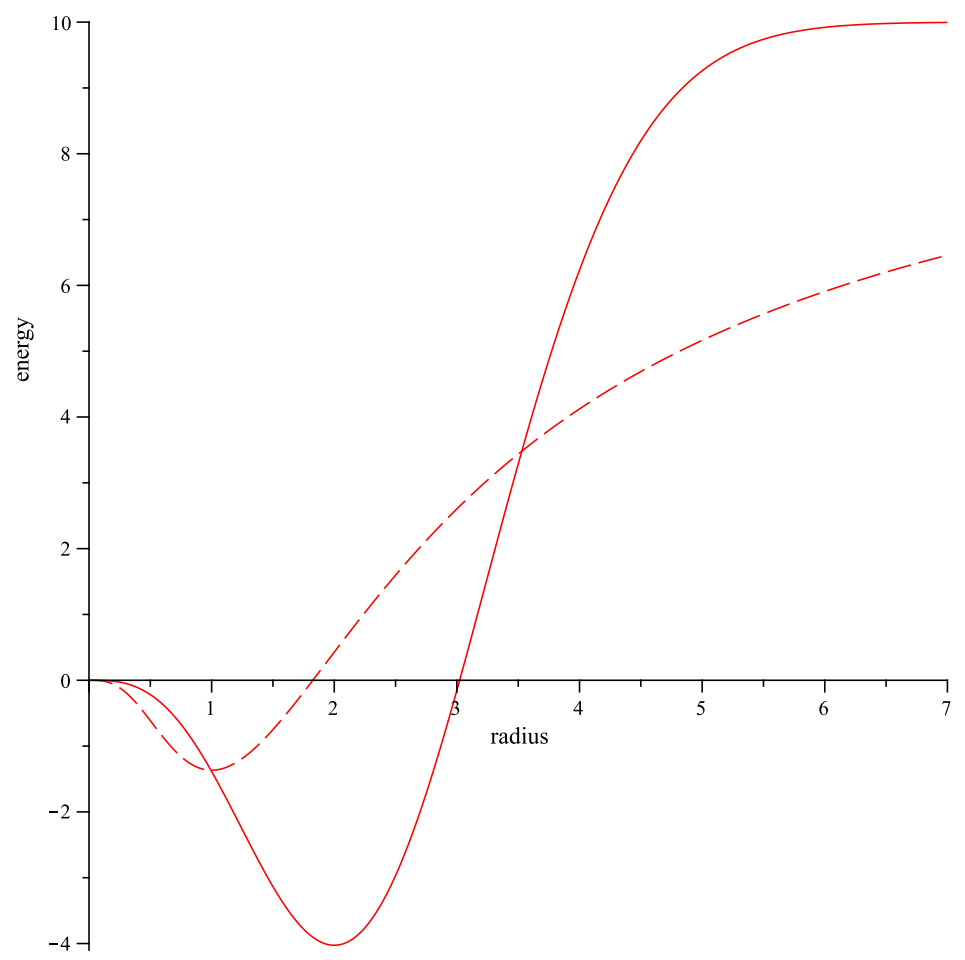

Figure 2: Energy versus radius. The solid line, corresponds to the Gaussian smearing and the dashed line, corresponds to the Lorentzian smearing. Other conditions for plotting the figure are similar to Fig. 1 We set $M=10.0$. For plotting the figure we set $\theta=\theta^{\prime}=1$. As can be seen from the figure, the energy on a holographic screen with radius $r_{0}$ is zero. In the case of $r<r_{0}$, the unusual feature, i.e. $E<0$, is again evident in both situations.

Finally, it should be noted that the value of $\sqrt{\theta}$ is directly associated with the noncommutative scale and is assuredly proportional to it. Nevertheless, the detailed nature of this correlation is not clearly described and would need a more exact framework to set it up. It is solely adequate to bear in mind that $\sqrt{\theta}$ is proportional to the length scale or inverse mass scale related to the noncommutative effects. The smallness of the scale would indicate that noncommutativity influences can be conceived just in excessive energy phenomena. In a general string theory context one could suppose that $\sqrt{\theta}$ would naturally not be far from the 4-dimensional Planck scale, $L_{P l}$. Most of the phenomenological investigations on noncommutative approaches have suggested that we live

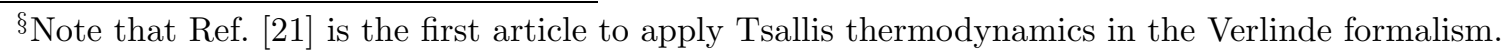


in a 4-dimensional spacetime and that the noncommutative energy scale is about $1-10$ $\mathrm{TeV}$ [22], accessible to colliders. Since, the minimal observable length is not precisely defined through deduction; therefore the scale is generally postulated as smaller than the typical scale of the standard model of particle physics, i.e. only less than $10^{-16} \mathrm{~cm}$. From another point of view, the fundamental Planck scale in models with extradimensions [23] can be neighboring current particle physics experiments [24], and it may be achieved in a $\mathrm{TeV}$ regime. Moreover, due to the appearance of possible extra spatial dimensions in the $\mathrm{TeV}$ range, the abundant creation of $\mathrm{TeV}$-scale black holes at colliders prove feasible [25]. Therefore it is acceptable to discuss that the properties of such TeV-scale black holes may be affected by noncommutativity influences, which are produced at a comparable scale. If the noncommutative energy scale is actually of order of the $\mathrm{TeV}$ range, then a direct probing of noncommutative physics may be possible for instance at the LHC, ILC, CLIC or some other future particle colliders.

\section{Summary and Conclusion}

In this article, we have discussed some aspects of Verlinde's proposal in the presence of noncommutative Schwarzschild black hole based on Gaussian-smeared mass and Lorentziansmeared mass distributions. The important difference between the two distributions is apprehended to occur around $r_{0}$, where there exists mostly the reactiveness to noncommutative effects and the precise form of the matter profile. Nevertheless, both mass distributions prepare the same quantitative aspects for the entropic force. Among these aspects, it is worth of emphasizing: (i) the fact that the gravitational and the inertial masses seem to be separate contravenes the equivalence principle of general relativity. However, one can come to this conclusion that gravitational and inertial masses are not necessarily distinctive, but the entire noncommutativity approach in gravity may be incorrect. In fact, there is a no-go theorem for noncommutative geometry inspired Schwarzschild black hole in Verlinde's scenario to entropic force. Otherwise, one would contravene the equivalence principle. (ii) at Planck scales either our analysis is not the suitable one, or non-extensive statistics should be applied. In fact, the comparison of the results obtained by the Gaussian profile with the results that we obtained by the Lorentzian profile, leads to two important conclusions: either we really cannot place a total trust in the noncommutative effects with the Gaussian, Lorentzian and some other 
cases of the smeared mass distribution at the regions with the order of Planck length, or we actually should have some misgivings in the results of standard thermodynamics at quantum gravity level which the origin of this proposition may imaginably be a result of the fractal nature of spacetime at very short distances. These ambiguities seem to be good factors for a possible experimental verification of Newton's law at very short distances in the future. Indeed, at present we do not know which of these decisions are reliable. However, we can always be enthusiastic about the possibility of an experimental breakthrough.

\section{References}

[1] S. W. Hawking, Comm. Math. Phys. 43, 199 (1975).

[2] T. Jacobson, Phys. Rev. Lett. 75, 1260 (1995) arXiv:gr-qc/9504004.

[3] T. Padmanabhan, Mod. Phys. Lett. A 25, 1129 (2010) arXiv:0912.3165.

[4] E. P. Verlinde, JHEP 1104, 029 (2011) arXiv:1001.0785.

[5] There is a large literature on this subject; see for example, R. G. Cai, L. M. Cao and N. Ohta, Phys. Rev. D 81, 061501 (2010) arXiv:1001.3470; M. Li and Y. Wang, Phys. Lett. B 687, 243 (2010) arXiv:1001.4466]; D. A. Easson, P. H. Frampton and G. F. Smoot, Phys. Lett. B 696, 273 (2011) arXiv:1002.4278]; C. Gao, Phys. Rev. D 81, 087306 (2010) arXiv:1001.4585]; J. Kowalski-Glikman, Phys. Rev. D 81, 084038 (2010) arXiv:1002.1035]; R. G. Cai, L. M. Cao and N. Ohta, Phys. Rev. D 81, 084012 (2010) [arXiv:1002.1136]; Y. Tian and X. Wu, Phys. Rev. D 81, 104013 (2010) arXiv:1002.1275]; Y. S. Myung and Y. W. Kim, Phys. Rev. D 81, 105012 (2010) arXiv:1002.2292]; R. A. Konoplya, Eur. Phys. J. C 69, 555 (2010) arXiv:1002.2818; W. G. Paeng and M. Rho, Mod. Phys. Lett. A 25, 399 (2010) arXiv:1002.3022; R. Banerjee and B. R. Majhi, Phys. Rev. D 81, 124006 (2010) arXiv:1003.2312; Y. S. Myung, Eur. Phys. J. C 71, 1549 (2011) arXiv:1003.5037; B. Koch, AIP Conf. Proc. 1232, 313 (2010) arXiv:1004.2879]; J. R. Mureika and R. B. Mann, Mod. Phys. Lett. A 26, 171 (2011) arXiv:1005.2214]; P. Nicolini, Phys. Rev. D 82, 044030 (2010) arXiv:1005.2996]; F. R. Klinkhamer, Class. Quant. Grav. 28, 125003 (2011) arXiv:1006.2094]; R. Percacci and G. P. Vacca, Class. Quant. Grav. 27, 245026 (2010) arXiv:1008.3621]; V. V. Kiselev and S. A. Timofeev, Mod. 
Phys. Lett. A 26, 109 (2011) arXiv:1009.1301]; A. Kobakhidze, Phys. Rev. D 83, 021502 (2011) arXiv:1009.5414; H. Sahlmann, Class. Quant. Grav. 28, 015006 (2011) arXiv:1010.2650]; Y. F. Cai and E. N. Saridakis, Phys. Lett. B 697280 (2011) arXiv:1011.1245]; S. H. Hendi and A. Sheykhi, Phys. Rev. D 83, 084012 (2011) arXiv:1012.0381]; I. Sakalli, Int. J. Theor. Phys. 50, 2426 (2011) arXiv:1103.1728; K. Nozari and S. Akhshabi, Phys. Lett. B 700, 91 (2011) [arXiv:1104.4849]; T. Qiu and E. N. Saridakis, arXiv:1107.1013; M. Visser, JHEP 1110, 140 (2011) arXiv:1108.5240]; K. Nozari, P. Pedram and M. Molkara, arXiv:1111.2204].

[6] See for instance, S. Hossenfelder, arXiv:1003.1015]; J. J. Roveto and G. Munoz, arXiv:1201.2475].

[7] P. Nicolini, A. Smailagic and E. Spallucci, Phys. Lett. B 632, 547 (2006) arXiv:gr-qc/0510112.

[8] A. Smailagic and E. Spallucci, J. Phys. A 36, L467 (2003) arXiv:hep-th/0307217; J. Phys. A 36, L517 (2003) arXiv:hep-th/0308193]; J. Phys. A 37, 7169 (2004) arXiv:hep-th/0406174]; E. Spallucci, A. Smailagic and P. Nicolini, Phys. Rev. D 73, 084004 (2006) arXiv:hep-th/0604094; R. Banerjee, B. Chakraborty, S. Ghosh, P. Mukherjee and S. Samanta, Found. Phys. 39, 1297 (2009) arXiv:0909.1000]; R. Banerjee, S. Gangopadhyay and S. K. Modak, Phys. Lett. B 686, 181 (2010) arXiv:0911.2123; L. Modesto and P. Nicolini, Phys. Rev. D 81, 104040 (2010) arXiv:0912.0220]; P. Nicolini and M. Rinaldi, Phys. Lett. B 695, 303 (2011) arXiv:0910.2860.

[9] P. Nicolini, Int. J. Mod. Phys. A 24, 1229 (2009) arXiv:0807.1939].

[10] T. Padmanabhan, Phys. Rev. D 81, 124040 (2010) arXiv:1003.5665.

[11] K. Nozari and S. H. Mehdipour, Class. Quant. Grav. 25, 175015 (2008) arXiv:0801.4074; JHEP 03, 061 (2009) arXiv:0902.1945]; S. H. Mehdipour, Commun. Theor. Phys. 52, 865 (2009); Int. J. Mod. Phys. A 25, 5543 (2010) arXiv:1004.1255]; Phys. Rev. D 81, 124049 (2010) [arXiv:1006.5215]; Can. J. Phys. 90, 425 (2012) arXiv:1204.0143].

[12] Y. X. Liu, Y. Q. Wang and S. W. Wei, Class. Quant. Grav. 27, 185002 (2010) arXiv:1002.1062. 
[13] R. J. Adler, P. Chen and D. I. Santiago, Gen. Rel. Grav. 33, 2101 (2001) arXiv:gr-qc/0106080].

[14] M. Gasperini, Phys. Rev. D 38, 2635 (1988); Phys. Rev. D 39, 3606 (1989); G. Z. Adunas, E. Rodriguez-Milla and D. V. Ahluwalia, Phys. Lett. B 485, 215 (2000) arXiv:gr-qc/0006021]; Gen. Rel. Grav.33 183 (2001) arXiv:gr-qc/0006022]; J. R. Mureika, Phys. Rev. D 56, 2408 (1997) arXiv:hep-ph/9612391].

[15] D. Singleton, S. Wilburn, Phys. Rev. Lett. 107, 081102 (2011) arXiv:1102.5564].

[16] M. Duncan, R. Myrzakulov and D. Singleton, Phys. Lett. B 703, 516 (2011) arXiv:1103.1713.

[17] K. Nozari and S. H. Mehdipour, Chaos Solitons Fractals 39, 956 (2009) arXiv:hep-th/0610076].

[18] M. S. El Naschie, Chaos Solitons Fractals 22, 495 (2004).

[19] L. Nottale, Fractal space-time and microphysics: towards a theory of scale relativity, World Scientific, Singapore, (1993).

[20] C. Tsallis, Braz. J. Phys. 29, 1 (1999) arXiv:cond-mat/9903356; C. Tsallis and E. Brigatti, Continuum Mech. Thermodyn. 16, 223 (2004) arXiv:cond-mat/0305606].

[21] J. A. Neto, [arXiv:1101.2927].

[22] I. Hinchliffe, N. Kersting and Y.L. Ma, Int. J. Mod. Phys. A 19, 179 (2004) arXiv:hep-ph/0205040]; J. L. Hewett, F. J. Petriello and T. G. Rizzo, Phys. Rev. D 66, 036001 (2002) arXiv:hep-ph/0112003]; J. M. Conroy, H.J. Kwee and V. Nazaryan, Phys. Rev. D 68, 054004 (2003) [arXiv:hep-ph/0305225]; T. Ohl and J. Reuter, Phys. Rev. D 70, 076007 (2004) arXiv:hep-ph/0406098.

[23] I. Antoniadis, Phys. Lett. B 246, 377 (1990); J. D. Lykken, Phys. Rev. D 54, 3693 (1996) arXiv:hep-th/9603133]; E. Witten, Nucl. Phys. B 471, 135 (1996) arXiv:hep-th/9602070; N. Arkani-Hamed, S. Dimopoulos and G. R. Dvali, Phys. Lett. B 429, 263 (1998) arXiv:hep-ph/9803315; I. Antoniadis, N. Arkani-Hamed, S. Dimopoulos and G. R. Dvali, Phys. Lett. B 436, 257 (1998) arXiv:hep-ph/9804398; L. Randall and R. Sundrum, Phys. Rev. Lett. 
83, 3370 (1999) arXiv:hep-ph/9905221); Phys. Rev. Lett. 83, 4690 (1999) arXiv:hep-th/9906064.

[24] E. G. Adelberger, J. H. Gundlach, B. R.Heckel, S. Hoedl and S. Schlamminger, Prog. Part. Nucl. Phys. 62, 102 (2009); V. B. Bezerra, G. L. Klimchitskaya, V. M. Mostepanenko and C. Romero, Phys. Rev. D 81, 055003 (2010) [arXiv:1002.2141].

[25] T. Banks and W. Fischler, arXiv:hep-th/9906038]; S. Dimopoulos and G. Landsberg, Phys. Rev. Lett. 87, 161602 (2001) arXiv:hep-ph/0106295]; S. B. Giddings and S. Thomas, Phys. Rev. D 65, 056010 (2002) arXiv:hep-ph/0106219]; P. Kanti, Int. J. Mod. Phys. A 19, 4899 (2004) arXiv:hep-ph/0402168; T. G. Rizzo, JHEP 0506, 079 (2005) arXiv:hep-ph/0503163. 